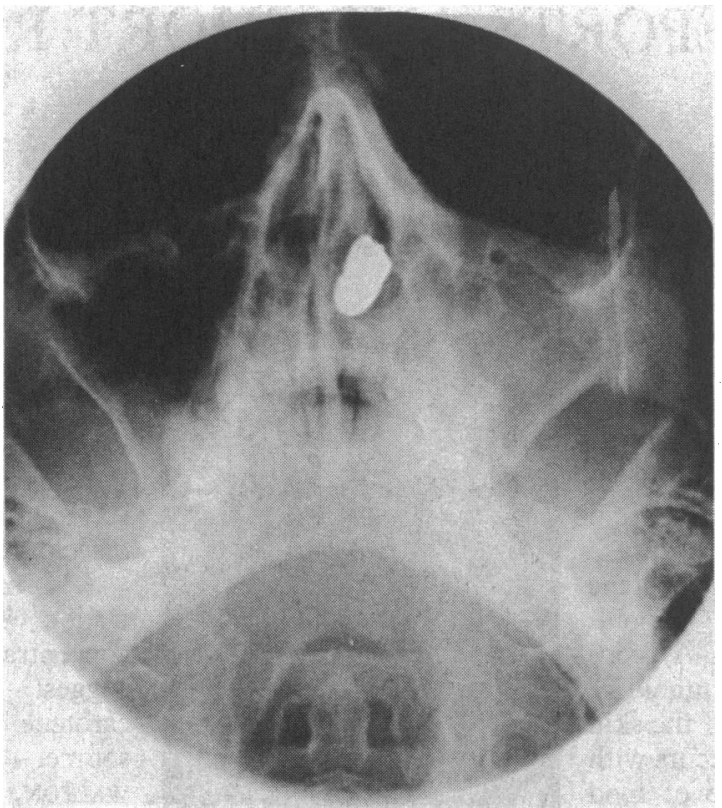

\title{
An unusual nasal foreign body
}

A 30 year old man was referred to this department with an intractable obstruction in one nasal cavity and catarrh of indeterminate duration. Inspection of his nose showed that one nasal cavity was normal; the other, however, was filled by a large polyp and mucopus. An $x$ ray film of the paranasal sinuses showed a metallic radio-opaque area in the nasal cavity resembling a bullet (figure). The man's nose was examined under general anaesthesia. Many polyps and a bullet were removed and the ipsilateral maxillary antrum drained.

Apparently he had sustained multiple gunshot wounds to the chest and thighs about 10 years previously. He had not had facial injuries so the bullet must have entered through the anterior nostril. There was no damage to the nasal septum or the turbinal bones, and at review his nose appeared completely normal. - C WASSON, otolaryngology department, Ulster Hospital, Northern Ireland

\section{Erosive pustular dermatosis of the scalp presenting as herpes zoster}

Erosive pustular dermatosis of the scalp was first described in 1979.' It consists of sterile pustules which lead to scarring alopecia. Two elderly women recently presented with a similar history: oozing and crusting of the scalp had been present for months (figure). Virological and bacteriological tests on specimens yielded negative results, and an erroneous diagnosis of herpes zoster resulted in inappropriate treatment.

Herpes zoster usually heals within four to six weeks. If the lesions persist the initial diagnosis was probably not correct and erosive pustular dermatosis of the scalp should be considered instead. Though lesions may seem to be confined to a single dermatome, they gradually extend to much larger areas of the scalp and extensive hair loss occurs. The exact nature of this disorder is uncertain, but the rapid response to topical steroid preparations suggests that it is not primarily infective in origin. - L SHALL, D SHUTTLEWORTH, department of dermatology, University Hospital of Wales, Cardiff CF4 4XN

Pye RJ, Peachey RDG, Burton JL. Erosive pustular dermatosis of the scalp. Brf Dermatol 1979; 100:559-66.

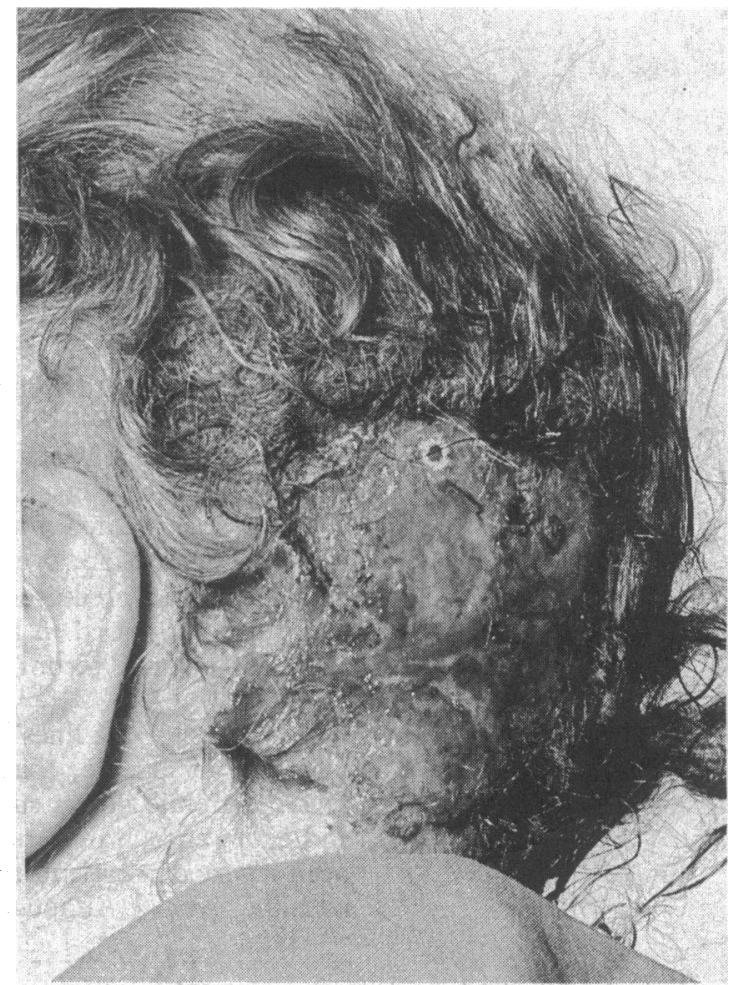

\section{Seamstress's finger: a cause of Heberden's nodes}

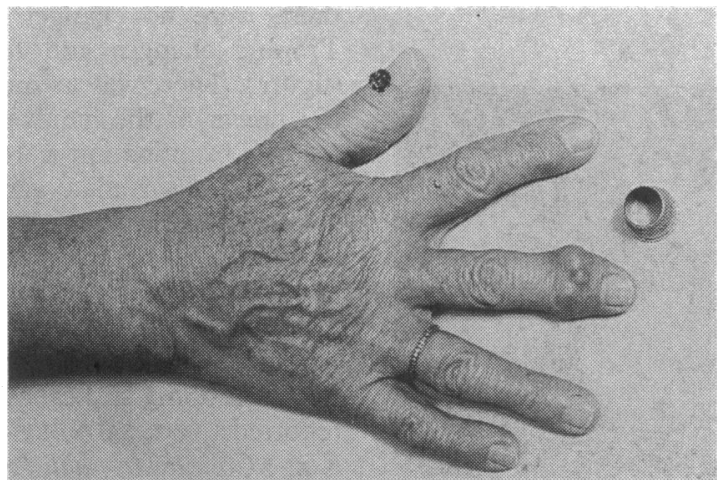

A retired seamstress presented with swellings around the distal interphalangeal joint of her right middle finger which were indistinguishable from classical Heberden's nodes. There was no evidence of osteoarthritis in any other joints and her serum urate concentration was normal. For more than 60 years she had used a seamstress's open ended thimble, which she jammed hard on to the affected finger. Driving a needle against resistance requires considerable lateral force to be exerted on the thimble. We believe that repeated abnormal stress applied to the distal interphalangeal joint was the cause of this case of Heberden's nodes. W H TURNER, JACK COLLIN, Nuffield department of surgery, Fohn Radcliffe Hospital, Oxford OX $39 D U$ 